\title{
Astrocyte-Associated Fibronectin Is Critical for Axonal Regeneration in Adult White Matter
}

\author{
Veronica J. Tom, ${ }^{1}$ Catherine M. Doller, ${ }^{1}$ Alfred T. Malouf, ${ }^{1,2}$ and Jerry Silver ${ }^{1}$ \\ Departments of ${ }^{1}$ Neurosciences and ${ }^{2}$ Pediatrics, Case Western Reserve University, Cleveland, Ohio 44106
}

\begin{abstract}
Although it has been suggested that astroglia guide pioneering axons during development, the cellular and molecular substrates that direct axon regeneration in adult white matter have not been elucidated. We show that although adult cortical neurons were only able to elaborate very short, highly branched, dendritic-like processes when seeded onto organotypic slice cultures of postnatal day 35 (P35) rat brain containing the corpus callosum, adult dorsal root ganglion (DRG) neurons were able to regenerate lengthy axons within the reactive glial environment of this degenerating white matter tract. The callosum in both P35 slices and adult rat brain was rich in fibronectin, but not laminin. Furthermore, the fibronectin was intimately associated with the intratract astrocytes. Blockade of fibronectin function in situ with an anti-fibronectin antibody dramatically decreased outgrowth of DRG neurites, suggesting that fibronectin plays an important role in axon regeneration in mature white matter. The critical interaction between regrowing axons and astroglial-associated fibronectin in white matter may be an additional factor to consider when trying to understand regeneration failure and devising strategies to promote regeneration.
\end{abstract}

Key words: astrocyte; astroglia; callosum; dorsal root ganglion; DRG; fibronectin; regeneration; slice

\section{Introduction}

The guiding role of astroglia in axonal navigation was initially proposed after the observation that oriented glial structures presage the route of pioneering populations of axons during embryonic development (Silver et al., 1993; Pires-Neto et al., 1998; Braga de Souza and Lent, 2004). Because astrocytes lose their axon growth-stimulating potency during aging (Smith et al., 1990), the role of mature astroglia in directing axon regeneration in adult white matter was not considered until adult sensory neurons microtransplanted into the callosum (Davies et al., 1997) and dorsal columns of the spinal cord (Davies et al., 1999) displayed robust regeneration in both undamaged and lesioned white matter. What might be the cellular and molecular substrates that mediate this regrowth potential in the purportedly inhibitory environment of CNS myelin (Cadelli and Schwab, 1991; Filbin, 2003)? Because the pattern of regenerating axons in vivo was mostly parallel to the host tract, it was suggested that such rapid growth is stimulated, at least in part, by the longitudinally organized intratract astrocytes, even though they had entered into some form of their reactive state. Although the haptotactic axonal guidance role of astrocytes has been demonstrated

Received June 1, 2004; revised Sept. 2, 2004; accepted Sept. 3, 2004.

This work was supported by National Institute of Neurological Disorders and Stroke Grants NS25713 (J.S.) and NS41383 (A.T.M.), the Daniel Heumann Fund, and the Brumagin Memorial Fund. We are grateful to Maryanne Pendergast for her excellent technical assistance with the confocal microscope and Dr. John Houlé for generously providing the computer software used for some of the quantification.

Correspondence should be addressed to Dr. Jerry Silver, Case Western Reserve University, Department of Neurosciences, 10900 Euclid Avenue, Cleveland, 0H 44106. E-mail: jxs10@cwru.edu.

DOI:10.1523/JNEUROSCI.2120-04.2004

Copyright $\odot 2004$ Society for Neuroscience $\quad$ 0270-6474/04/249282-09\$15.00/0 conclusively in vitro (Noble et al., 1984; Smith et al., 1990; Biran et al., 2003), their potential to foster regenerative growth in vivo has yet to be elucidated.

In addition to their patterned geometry, astrocytes are thought to provide both growth-promoting and growthinhibiting molecules, the balance of which within the environment of navigating axons can either attract or deflect outgrowth. Various growth-promoting molecules, including laminin (Liesi, 1985; Liesi and Silver, 1988), N-cadherin (Neugebauer et al., 1988; Tomaselli et al., 1988), neural cell adhesion molecule (NCAM) (Neugebauer et al., 1988; Smith et al., 1990), and fibronectin (Price and Hynes, 1985; Liesi et al., 1986; Matthiessen et al., 1989) have been suggested to allow astrocytes to promote axonal outgrowth during development. On the contrary, inhibitory proteoglycans associated with glial boundaries during development (Snow et al., 1990; Gonzalez et al., 1993; Steindler, 1993) and glial scarring in the lesion penumbra (Davies et al., 1999; Fitch et al., 1999; Asher et al., 2000; Jones et al., 2003, Tom et al., 2004) prevent axons from growing further.

To better understand the cellular and molecular mechanisms that foster robust regeneration of adult axons through mature white matter, we developed an in situ slice culture system to study axon regeneration in a living, easily manipulated, threedimensional environment. Just as they do after microtransplantation in vivo, dissociated adult dorsal root ganglion (DRG) neurons seeded onto postnatal day 35 (P35) coronal organotypic slice cultures of cortex containing the corpus callosum grow extremely well in this heavily myelinated white matter tract. Contrary to previous findings, the fully mature callosum is rich in astrocytic-associated fibronectin that appears to be critical for sensory axon outgrowth. Our findings suggest that astroglialassociated fibronectin in adult white matter tracts may be a crit- 
ical extracellular molecule (ECM) that is provided to severed axons in the CNS. Whether adult axons are capable of responding maximally to this readily available extracellular substrate may be an additional factor to consider when trying to understand regeneration failure as well as devising strategies to promote regeneration.

\section{Materials and Methods}

$D R G$ dissociation. DRG neurons were obtained as described previously (Davies et al., 1997). Briefly, adult green fluorescent protein (GFP)expressing transgenic mice were anesthetized and decapitated. After the spinal column was dissected, the DRGs were removed ( $\sim 30$ ganglion from the cervical, thoracic, and lumbar segments of the spinal cord), the roots were trimmed, and the ganglia were incubated in dispase-collagenase for $1.5 \mathrm{hr}$ at room temperature (RT). Afterward, the ganglia were washed several times in calcium and magnesium-free-HBSS (CMF) and then gently triturated. The cell suspension was spun several times to help remove satellite glia $(\sim 370 \times g)$ and resuspended in Neurobasal A/B27 at $\sim 100$ cells per microliter.

Cortical neuron dissociation. Adult cortical neurons were obtained using a modified method of Brewer (1997) that is specifically designed for the culture of adult CNS tissues. Adult GFP + mice were anesthetized and decapitated. The brains were quickly dissected out, and the cortices were removed. The tissue was quickly minced and incubated in papain (2 $\mathrm{mg} / \mathrm{ml}$; Worthington, Freehold, NJ) for $30 \mathrm{~min}$ at $37^{\circ} \mathrm{C}$. The tissue was rinsed several times in $\mathrm{CMF}$ and then gently triturated. The suspension was spun in a density gradient to enrich for neurons, and the appropriate fraction was isolated, rinsed several times, and resuspended in Neurobasal A/B27 at $\sim 100$ cells per microliter.

Organotypic slice culture. P35 Sprague Dawley rats were anesthetized and decapitated. The brains were quickly removed from the skull, blocked, and glued onto a sterile pedestal. Coronal slices $(200 \mu \mathrm{m})$ containing the corpus callosum were cut in ice-cold CMF. Each slice was then placed on a $25 \mathrm{~mm}$ tissue culture insert with a $0.02 \mu \mathrm{m}$ pore size (Nunc, Naperville, IL) in Neurobasal A/B27 (Invitrogen, San Diego, CA) with $100 \mu \mathrm{M}$ CNQX and $100 \mu \mathrm{M}$ AP-5 to block glutamate receptormediated toxicity (Hafidi et al., 1999). The next day, $\sim 150$ freshly dissociated DRG neurons were seeded onto the corpus callosum. Two hours later, $3 \times 2 \mu \mathrm{l}$ of rabbit IgG (RIgG) $(25.5 \mathrm{ng} / \mathrm{ml}$ as a control for antifibronectin; $10 \mu \mathrm{g} / \mathrm{ml}$ as a control for anti-laminin; Chemicon, Temecula, CA), rabbit anti-fibronectin $(25.5 \mathrm{ng} / \mathrm{ml}$ or 1:200; Dako, High Wycombe, UK), or rabbit anti-laminin $(10 \mu \mathrm{g} / \mathrm{ml}, 1: 1000$; Biomedical Technologies, Stoughton, MA) were spotted all over the region of the corpus callosum of alternate sections, as done previously (Butler et al., 2004). Although there have been other studies using various fibronectin antibodies to block fibronectin function (Donaldson et al., 1985; Smith et al., 1990; Moursi et al., 1996; Guo et al., 2001), to the best of our knowledge, this is the first one to use this particular polyclonal anti-fibronectin antibody for that purpose. Rabbit IgG, anti-fibronectin, or anti-laminin was also added to the media. The media was changed and the antibodies were replenished every day.

Tissue culture. Coverslips were coated with poly-L-lysine $(1 \mathrm{mg} / \mathrm{ml})$ overnight, rinsed several times with sterile water, and allowed to dry. Some coverslips were then incubated with fibronectin $(10 \mu \mathrm{g} / \mathrm{ml})$ or laminin $(10 \mu \mathrm{g} / \mathrm{ml})$. The remaining coverslips were not coated with anything other than poly-lysine. Freshly dissociated DRG neurons were plated onto the coverslips. Two hours after cell plating, cultures on the fibronectin-coated coverslips were treated with either anti-fibronectin or rabbit $\operatorname{IgG}$ (concentrations as mentioned above), and cultures on the laminin-coated coverslips were treated with either anti-laminin or rabbit IgG. To control for antibody-induced toxicity, cultures on the polylysine coverslips were treated similarly.

Cortical astrocyte dissociation. Astrocytes were obtained from P1 Sprague Dawley rats. The pups were decapitated, and the cortices were removed, finely minced, and treated with $0.1 \%$ trypsin in EDTA. Cells were rinsed in serum-containing media, gently spun down, and then seeded onto poly-lysine-coated flasks in DMEM/F12 with 10\% FBS. Four hours later, the media was changed after the flasks were shaken to remove nonadherent cells. The astrocytes were maintained for 5 weeks, when they were gently trypsinized from the surface of the flasks, rinsed in fresh media, and plated on poly-lysine-coated coverslips at a density of $75,000-100,000$ cells per coverslip. The next day, RIgG, anti-fibronectin, or anti-laminin was added to the media. The cultures were maintained for an additional $3 \mathrm{~d}$ before fixation in $4 \%$ paraformaldehyde.

Immunohistochemistry. Organotypic slice cultures were immersion fixed in $4 \%$ paraformaldehyde in 0.1 м PBS for $2 \mathrm{hr}$. Cultures on coverslips were fixed in $4 \%$ paraformaldehyde for $30 \mathrm{~min}$. In addition, rats were transcardially perfused with ice-cold $4 \%$ paraformaldehyde in PBS, and the brains were dissected out. After the tissue was postfixed in $4 \%$ paraformaldehyde overnight at $4^{\circ} \mathrm{C}, 50 \mu \mathrm{m}$ sections were cut on a vibratome. After several rinses in PBS, coverslip cultures were blocked with $5 \%$ NGS, $0.1 \%$ BSA plus $0.1 \%$ Triton X-100 in PBS for $1 \mathrm{hr}$ at RT before they were incubated in primary antibody overnight at $4^{\circ} \mathrm{C}$. Slice cultures and brain sections were blocked with 3\% NGS, 3\% BSA, 0.1\% DMSO, and $0.1 \%$ Triton X-100 (if the antigens were intracellular) in PBS for $1 \mathrm{hr}$ at RT before they were incubated in primary antibody overnight at $4^{\circ} \mathrm{C}$. The primary antibodies used were rabbit anti-GFP (1:1000; Chemicon), mouse anti-GFAP (1:500; Sigma, St. Louis, MO), mouse anti-myelin (MAB328, 1:1000; Roche Products, Hertfordshire, UK), mouse anti- $\beta$ tubulin type III (1:1000; Sigma), rabbit anti-fibronectin (1:400; Dako), and rabbit anti-laminin (1:1000; Biomedical Technologies). The cultures were rinsed in PBS and incubated in the appropriate secondary antibody either for $1.5 \mathrm{hr}$ at RT (coverslips) or overnight at $4^{\circ} \mathrm{C}$ (slice cultures and sections). They were rinsed again in PBS, mounted in Citifluor (Ted Pella), coverslipped, and sealed with nail polish. Specimens were examined with a Leitz Orthoplan 2 microscope and a Zeiss (Oberkochen, Germany) LSM 410 confocal microscope.

Quantification. To quantify axonal outgrowth on fibronectin- or laminin-coated coverslips treated with RIgG, rabbit anti-fibronectin, or rabbit anti-laminin, montaged images were taken of semirandomly chosen neurons ( $n=12$ per group) maintained for $3 \mathrm{~d}$ in culture and stained for $\beta$-tubulin III. The neurons were chosen semirandomly in the sense that they were viewed randomly. Neurons that were determined to be too close to a neighboring neuron (i.e., they contained neurites that were intertwined with each other) were discarded, and another was viewed. The longest neurite per neuron was measured (MetaMorph), and the lengths were analyzed statistically using a Student's $t$ test for statistical relevance.

To quantify outgrowth of neurons attached to either gray or white matter of the slices, confocal images were taken of the corpus callosum and the gray matter just adjacent to the callosum ( $n=4$ slices). Montages were made from the images, and all individual neurites that could be followed were digitally traced by hand using a consistent brush thickness that was approximately the width of an average neurite (Photoshop). The tracings alone were quantified for pixel area (MetaMorph), which was then divided by the number of cells extending neurites on gray or white matter to get a neurite length per cell average, which accounts for any differences in the numbers of cells that have attached on gray versus white matter. This process also eliminates any subtle differences in neurite width so that the only variable is the length of the neurites. These pixel area averages were analyzed using a Student's $t$ test for statistical relevance.

Outgrowth of neurites on white matter of slices treated with RIgG, anti-fibronectin, or anti-laminin was quantified similarly. Confocal images were taken of areas of the corpus callosum containing GFP+ neurites in RIgG-treated ( $n=10$, control for anti-fibronectin; $n=5$, control for anti-laminin), anti-fibronectin-treated $(n=10)$, and anti-laminintreated $(n=5)$ cultures. Montages were made from the images, and individual neurites were digitally traced using a consistent brush thickness that was approximately the width of an average neurite (Photoshop). The tracings were quantified for pixel area (MetaMorph), which was then divided by the number of cells (on the order of approximately two to three dozen per slice) that extended neurites to get a cell average. These pixel area averages were analyzed using a Student's $t$ test for statistical relevance. 


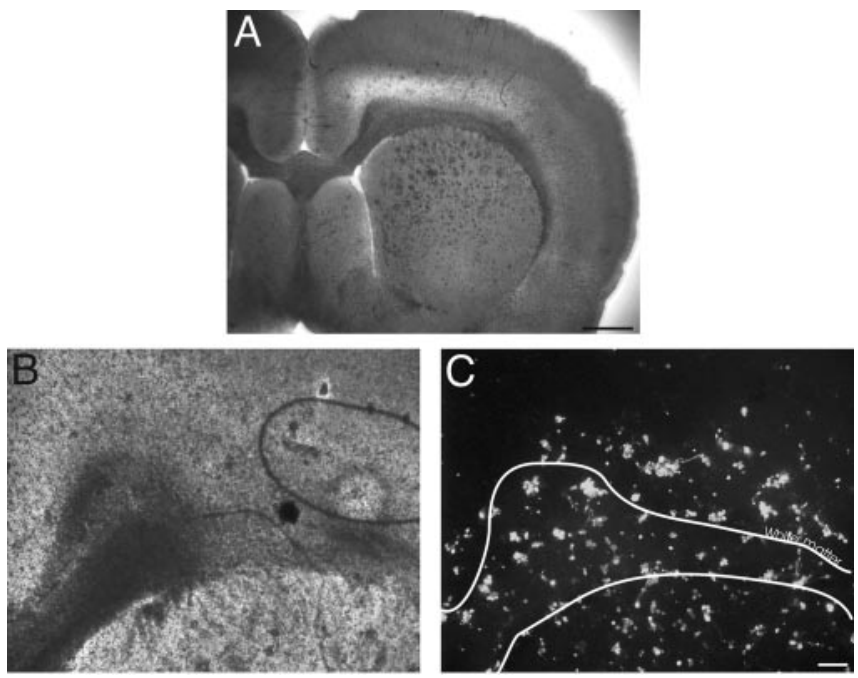

Figure 1. Dissociated adult DRGs attach to organotypic slices containing white matter. $A$, Low-magnification bright-field image of an organotypic coronal slice containing the white matter tract the corpus callosum that had been in culture for $4 \mathrm{~d}$. B, C, Dissociated GFP + DRGs were seeded onto the callosal region of 1 DIV slices and fixed and stained at 4 DIV. The brightfield image of the slice is shown in $B$. The fluorescent image of the GFP + neurons is shown in C. The white matter tract is denoted by the white lines. Note that many neurons were able to adhere to the white matter. Scale bars: $A, 500 \mu \mathrm{m} ; B, C, 200 \mu \mathrm{m}$.

\section{Results}

Robust regeneration of adult sensory neurons on the living corpus callosum in culture

Coronal organotypic slices of the cerebrum from P35 rats (Fig. $1 A, B$, shown in bright-field at low magnification) were able to support the adherence of adult sensory neurons, especially in the region that included the callosum (Fig. $1 C$ ). Moreover, the attached neurons were able to regenerate lengthy axons only within GFAP + astrocyte-rich regions (Fig. 2 A, arrowhead; GFAP in red, $\mathrm{GFP}+$ neurites in green) (see below for a quantification of axonal outgrowth). The sensory neurons attached less well to gray matter. When they did, they elaborated very short neurites (Fig. $2 B$, arrow, green). This significant difference in neurite outgrowth from neurons attached to either gray or white matter is quantified in Figure $2 E\left({ }^{\star} p<0.005\right)$. The P35 gray matter, unlike the callosum, after $4 \mathrm{~d}$ in culture was filled with necrotic debris and devoid of any GFAP+ astrocytes, suggesting that the gray matter astrocytes either had died or (albeit unlikely) had dedifferentiated into a non-GFAP-expressing state.

\section{Adult cortical neurons regenerate poorly on the corpus callosum in organotypic slices}

Although the corpus callosum provided a highly growthsupportive environment for adult sensory neurons, it turned out to be a poor substrate for the outgrowth of adult cortical neurons. Adult GFP+ cortical neurons (Fig. $3 A, C, D, F$, green) seeded on the white matter tract were able to generate highly branched processes; however, they were relatively short, and there was never a clear-cut lengthy axon. Every process that extended from the cell body contained MAP-2, a dendritic marker (Fig. $3 B, C, E, F$, red).

Sensory axon regeneration on the callosum occurs despite the presence of degenerating myelin

There have been a great number of studies suggesting that myelin and/or inhibitory myelin proteins released on the degeneration of lesioned axon tracts inhibit cell attachment and axonal regen- eration (for review, see Caroni et al., 1988; Cadelli and Schwab, 1991; Fournier and Strittmatter, 2001; Filbin, 2003; Spencer et al., 2003). In this assay, however, axon regeneration (at least from sensory neurons) was most robust in the myelin-rich corpus callosum. To determine whether cultured slices still contained myelin (i.e., disrupted myelin membranes), $1 \mathrm{~d}$ in vitro (1 DIV) slices (the time point when the dissociated neurons were seeded on the slice) or 4 DIV slices (the time point when neuritic outgrowth was assessed) were fixed and then stained for the presence of a myelin membrane antigen. The corpus callosi of slices from both time points were positive for this myelin marker (Figs. $4 A, B$ ). To get an overview of where the majority of the neurites grew (whether they extended on the surface of the slice or whether they were able to bury themselves below the surface), a z-series of confocal images of regenerating GFP + DRG neurites (Fig. $4 D$, green) within the GFAP + callosum was obtained (Fig. $4 D$, red). Although there were several neurites on the surface of the slice (Fig. 4D, arrowhead), the vast majority grew $\sim 20 \mu \mathrm{m}$ below the surface of the slice (Fig. $4 D$, yellow), where there was plenty of myelin debris present (Fig. 4C). Thus, in this model, robust regeneration of sensory axons occurs despite the presence of disrupted myelin.

\section{Neurites appear to crudely follow host astrocyte alignment}

In our assay, we found that the regenerated neurites followed the alignment of the host astrocytes only very generally. When the host astrocytes ran generally parallel in the corpus callosum (Fig. $5 A, B$, red), the neurites grew essentially in the same direction (Fig. 5A, $B$, arrowheads, green). When the host astrocytic processes extended radially in the cingulum (Fig. $5 C, D$, red), the neurites grew in that direction (Fig. $5 C, D$, arrows, green). This suggests that the orientation of the regenerating neurites is somewhat influenced by the alignment of the host astrocytes; however, it should be stressed that the neuritic alignment with astrocytes was only crudely aligned, at best, resulting in axon growth that meandered from one astrocytic process to another (Figs. $2 C, D$, $5 B$, asterisk).

\section{Fibronectin is expressed by callosal astrocytes}

To determine which growth-promoting factor might reside on the surface of the mature astrocyte, fresh, living slices were immediately fixed and stained for both laminin and fibronectin, two growth-promoting ECMs known to be made by immature astrocytes (Liesi et al., 1983, 1986; Liesi and Silver, 1988). Although it has been shown previously that cultured neonatal cortical astrocytes express laminin in vitro (Liesi et al., 1983), we found that in our P35 slice cultures, laminin staining was present only along blood vessels and virtually absent from the remainder of the corpus callosum (Fig. 6B). Although fibronectin was also expressed along the surface of blood vessels, we also found that it was highly expressed in the corpus callosum, well away from blood vessel territory (Fig. 6A). Differential expression of non-vesselassociated fibronectin (Fig. 6C), but not laminin (Fig. 6D), in the callosum was confirmed in sections of perfusion-fixed, adult rat brain. The callosal fibronectin appeared to be associated with $\mathrm{GFAP}+$ astrocytes (Fig. 6E-G, arrows).

\section{Polyclonal fibronectin and laminin antibodies block axonal outgrowth on their respective purified substrates}

To determine what role fibronectin plays in neurite outgrowth in white matter, we first needed to ensure that the antibody that we were using to detect fibronectin was function blocking. Dissociated DRGs were plated onto purified fibronectin or laminincoated coverslips, and after $2 \mathrm{hr}$ to allow for neuronal attachment, 


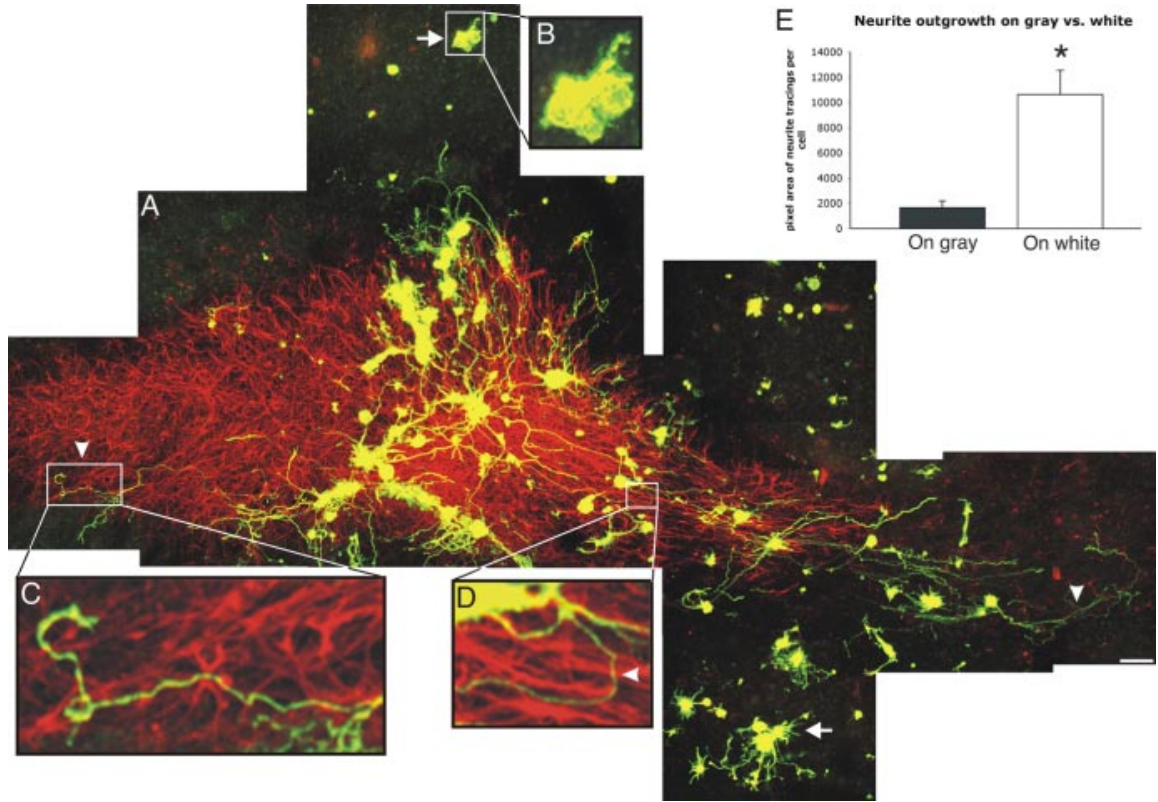

Figure 2. DRGs regenerate robustly on the corpus callosum. $A$, Confocal microscopy montage of GFP + DRGs (green) on the GFAP + astrocyte-rich corpus callosum (red). Insets are higher-magnification images of areas denoted by the white boxes. The DRGs were able to grow well on the callosum and extended long neurites ( $C$, arrowhead) after 3 DIV; however, neurons on gray matter, which was virtually devoid of GFAP + astrocytes, were able to extend only very short neurites ( $B$, arrows in main figure $A)$. Although much of the growth appeared to grossly follow the alignment of the host astrocyte network, there were numerous instances during which axons strayed and turned $(C, D$, arrowhead). $E$, Quantification of the disparity between outgrowth on gray and white matter. Neurites from DRGs seeded onto 1 DIV organotypic slice cultures and maintained for 3 DIV were traced digitally. Neurites originating from DRGs seeded on gray matter of the organotypic slice culture after 3 DIV were traced digitally and compared with those neurites that grew on the corpus callosum ( $n=4$ slices per group). The pixel areas of the tracings were quantified and analyzed statistically using a Student's $t$ test. There were significantly shorter neurites per neuron on gray matter than on white matter $\left({ }^{*} p<0.005\right)$. Scale bar, $100 \mu \mathrm{m}$.
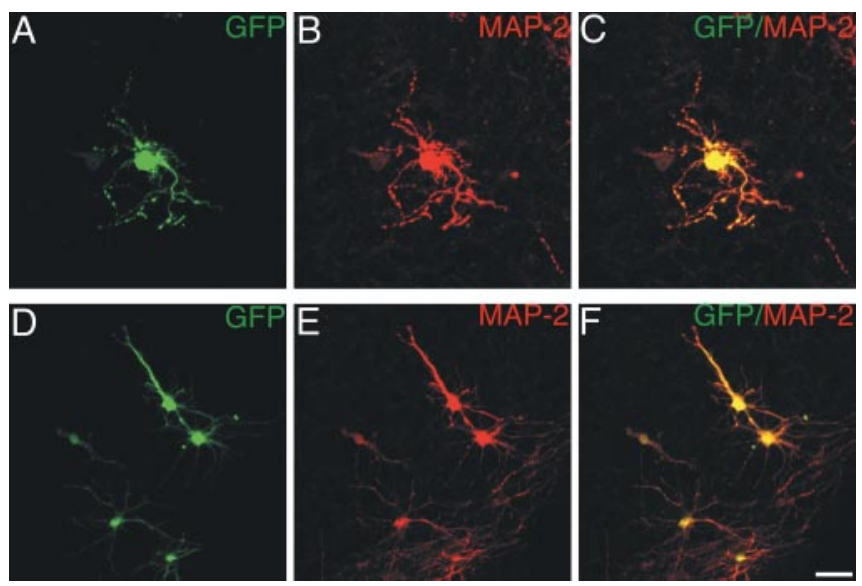

Figure 3. Cortical cells do not regenerate well on white matter. Adult cortical neurons seeded onto the corpus callosum of P35 organotypic slice cultures were double stained for GFP $(A, C, D, F$, green) and the dendritic marker MAP-2 $(B, C, E, F)$ after 8 DIV. Even after such a long time in culture, the neurons were able to extend only short neurites. Furthermore, all neurites were positive for MAP-2, suggesting that they were of a dendritic nature. Scale bar, $25 \mu \mathrm{m}$.

the polyclonal fibronectin antibody, the polyclonal laminin antibody, or RIgG was added to the media. Three days later the cultures were fixed, and neurite outgrowth was assessed. DRGs incubated with RIgG extended long neurites (Fig. 7 A, G); however, both the fibronectin antibody (Fig. $7 B, G)\left({ }^{*} p<0.00001\right)$ and the laminin antibody (Fig. $7 C, G)\left({ }^{* *} p<0.00000001\right)$ dramatically decreased neurite outgrowth on their appropriate substrates. To control for potential antibody-induced toxicity, RIgG (Fig. 7D), anti-fibronectin (Fig. 7E), or anti-laminin (Fig. $7 F$ ) was added to the media of DRG cultures plated on poly-lysine-coated coverslips. In all three cases, DRGs survived and were able to send out long processes. This suggests that the effect seen with the fibronectin and laminin antibodies on the fibronectin- and laminin-coated coverslips, respectively, is caused by a specific blockade of fibronectin- and lamininmediated outgrowth and not by antibodymediated toxicity.

\section{Fibronectin is critical for sensory axon regeneration in the corpus callosum} Because we had a function-blocking antibody against fibronectin, we used it in our slice culture model to determine how necessary fibronectin is for axonal outgrowth in white matter. The cultures were prepared as described above. Several hours after the dissociated DRGs were seeded onto the callosum, the fibronectin antibody was added both to the surface of the callosum and to the media. RIgG was added to alternate slices as a control. Although we were not able to visualize laminin in the corpus callosum via immunohistochemistry, we wanted to ensure that callosal laminin, which perhaps is present but difficult to visualize, is not playing a role in mediating outgrowth. Therefore, some slices were treated with either the function-blocking anti-laminin antibody or the appropriate concentration of RIgG as a control. Three days later, the slices were fixed and stained for GFP (for seeded neurons). GFP + DRGs on RIgG-treated slices appeared similar to those on untreated slices (Fig. $8 \mathrm{~A}$ ) and displayed robust outgrowth on the callosum. The laminin antibody also did not have an effect on neurite outgrowth on the callosum (Fig. 8D,E). Furthermore, treating slices with antisense against the $\gamma$ chain of laminin to inhibit laminin synthesis more broadly (Grimpe et al., 2002) also did not have an effect (data not shown). Although approximately the same number of neurons was seeded, relatively fewer DRGs remained attached to the antifibronectin-treated slices, and those that did attach had significantly shorter neurites (Fig. $8 \mathrm{~B}, E$ ). Interestingly, the longitudinal processes of the astrocytes in the anti-fibronectin-treated cultures appeared to be somewhat stunted (Fig. $8 C$, red), and the alignment of the astrocytic network did not appear to be as rectilinear as in RIgG and anti-laminin-treated cultures (Figs. 2, $5 A, B$, compare with Fig. $8 C$ ).

Although it is possible that the abnormal astrocytic patterning may be partially responsible for the decreased outgrowth in antifibronectin-treated cultures, it seems unlikely that a change in substrate geometry is the sole reason for the reduced outgrowth. Many DRG neurites in slices treated with anti-fibronectin were short, despite the fact that they had a significant length of contiguous astrocytic terrain remaining ahead of them (Fig. 8C, arrowhead, green). Such curtailed outgrowth in the presence of antibody suggests that axons were directly affected by a lack of astrocytic fibronectin. Total neurite outgrowth per cell was decreased by $\sim 50 \%$ in anti-fibronectin-treated cultures $\left({ }^{*} p<\right.$ 0.0001 ) (Fig. $8 E$ ), whereas there was no significant difference 
between anti-laminin- and RIgG-treated cultures. We are now planning a more complete study to determine the exact nature and substrate requirements of the processes that are made by cortical neurons in the slice environment, because these cells, unlike adult sensory neurons, appear to elaborate dendrites rather than axons.

To further ensure that the astrocytic phenotype change seen in the anti-fibronectin-treated slice cultures is not caused by antibody-induced glial toxicity, RIgG, anti-fibronectin, or antilaminin was added to confluent monolayers of astrocytes plated onto poly-lysine-coated coverslips. After $3 \mathrm{~d}$, the cultures were fixed and stained for GFAP. With all three treatments, astrocytes looked healthy and remained confluent, suggesting that none of the treatments are toxic to astrocytes (data not shown). Unlike the antibody-mediated change in astroglial shape in the organotypic slice cultures, astroglial morphology in dissociated culture was comparable in all treatment groups.

\section{Discussion}

To investigate what cellular and molecular factors promote robust sensory axon regeneration in white matter, dissociated adult DRG and cortical neurons were seeded onto organotypic slices containing the corpus callosum. Although cortical neurons extended very short, branched MAP-2+ neurites, sensory neurons regenerated long axons within the degenerating myelin along host astrocytes. Fibronectin was associated with astrocytes both in P35 slices and in the adult callosum, despite literature suggesting that fibronectin is expressed only transiently during development (Stewart and Pearlman, 1987; Rogers et al., 1989; Müller et al., 1995). Furthermore, blocking fibronectin significantly decreased the length of regenerating axons, suggesting that this molecule plays a critical role in stimulating regeneration along the astrocyte surface in white matter. Although a plethora of evidence in the literature suggests that astrocytes can express fibronectin (Price and Hynes, 1985; Liesi et al., 1986; Matthiessen et al., 1989; Smith et al., 1990; Pasinetti et al., 1993; Biran et al., 2003), we cannot yet rule out the possibility that other cell types, such as microglia-macrophages, which express fibronectin in certain populations (Adachi et al., 1988; Brown et al., 1993), or oligodendrocytes are additional sources of the molecule. It is also important to mention that the antibody did not fully prevent outgrowth, which may be caused by incomplete blockade of function because fibronectin contains multiple outgrowthpromoting domains (Rogers et al., 1985, 1987). To resolve this, it may be necessary to use both an arginine-glycine-aspartate peptide, a general antagonist for integrin receptors, and heparin, which will competitively bind the heparin-binding site of fibronectin. Moreover, we do not know whether other growth-promoting molecules in the slice (Gates et al., 1996), such as $\mathrm{N}$-cadherin and NCAM (Neugebauer et al., 1988; Tomaselli et al., 1988), or neuro-
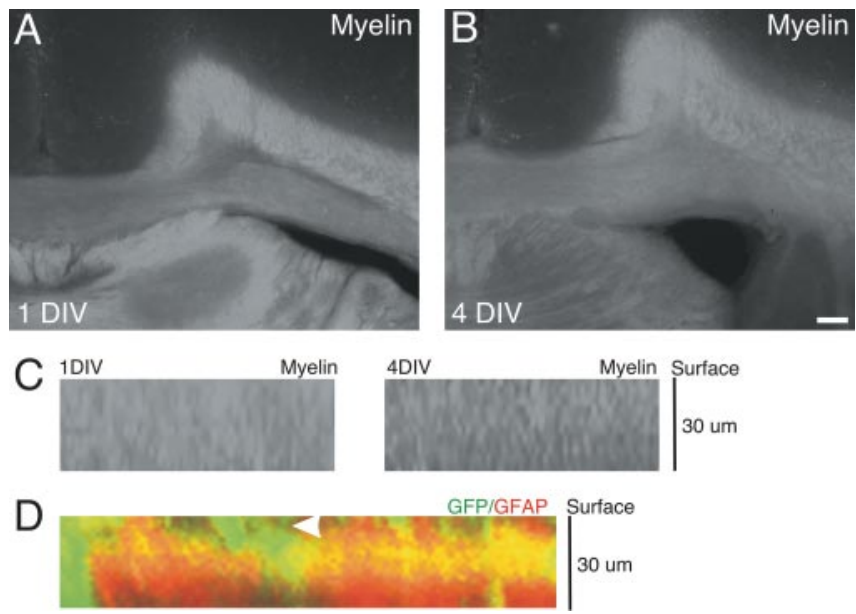

Figure 4. DRGs regenerate neurites despite the presence of myelin. $A, B$, An abundance of myelin was present in the corpus callosi of both 1 DIV slices (when neurons were seeded) and 4 DIV slices (when cultures were fixed and regeneration was assessed). C, D, Z-series of confocal microscope images revealed that although there were some GFP + neurites that remained on the surface of the slice ( $D$, green, arrowhead), the majority of regenerating neurites penetrated and grew $\sim 15-20 \mu \mathrm{m}$ below the surface of the slice ( $D$, yellow). There was plenty of myelin at this depth in the slice at both 1 and 4 DIV ( $C$, suggesting that the regenerating neurites were growing among myelin as well as callosal astrocytes (D, red). Scale bar: $A, B, 200 \mu \mathrm{m}$.
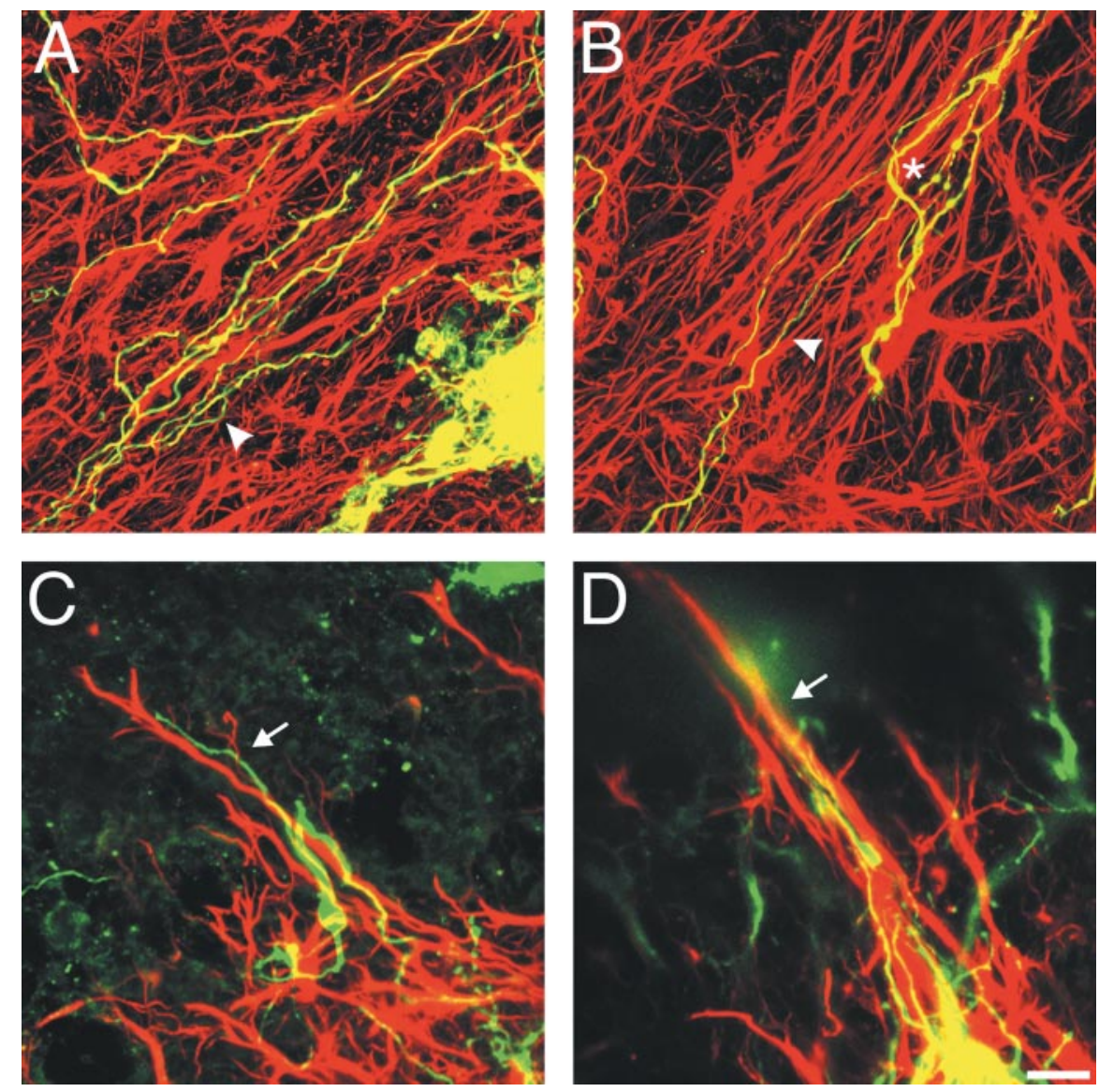

Figure 5. Regenerating neurites are only crudely aligned with host tract astrocytes. $A, B$, regenerating GFP + neurites (green) generally followed host GFAP + astrocytic processes in the callosum (red) and grew longitudinally (arrowheads). Alignment was not exact, however, and there were instances during which neurites wandered and jumped from one astrocytic process to another ( $B$, asterisk). $C, D$, Regenerating neurites (green) in the cingulum grew radially away from the callosum, apparently guided by the astrocytes that were present there (arrows). Scale bar, $25 \mu \mathrm{m}$. 

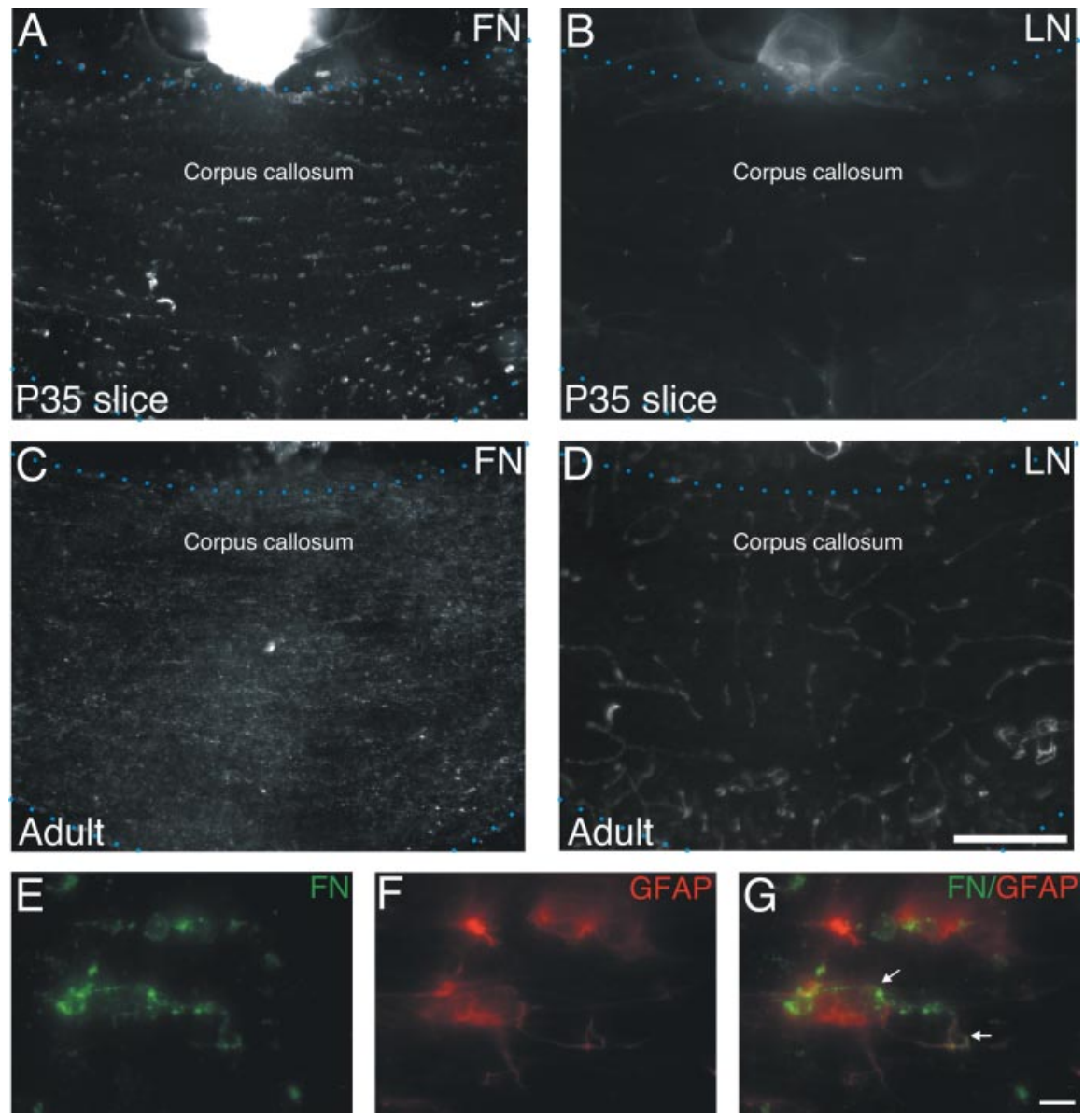

Figure 6. Fibronectin, not laminin, is expressed by astrocytes in the corpus callosum. $A, B$, Freshly fixed coronal slices stained for fibronectin $(A)$ and laminin $(B)$. Although laminin was visualized only along blood vessels, fibronectin was expressed all over the cortex, including the corpus callosum, demarcated by the blue dots. $C, D$, Perfused adult rat brain sections stained for fibronectin $(C)$ and laminin $(D)$. Again, the callosum was rich in fibronectin, whereas laminin was present only along blood vessels. $E-G, A$ GFAP + astrocyte in the callosum of a slice $(F, G$, red) was closely associated with fibronectin-expression patterns $(E, G$, arrows, green). Scale bars: $A-D, 50 \mu \mathrm{m} ; E-G, 5 \mu \mathrm{m}$.

trophic factors (Schwartz and Nishiyama, 1994; Dreyfus et al., 1999) also play a role in directing regeneration. It would be interesting to determine what other molecules are associated with callosal astrocytes and then block their activities to assess their roles in neurite outgrowth.

Using fresh organotypic slices satisfied our aim to develop an easily manipulated system to study axonal regeneration in white matter; however, the white matter within our in situ model may be different from that in vivo. Much of the slice is undergoing cell death, and although the astrocytes are clearly in a gliotic state, this type of reactive phenotype as well as the integrity of myelin in the slice may be quite different from that found in vivo, even after an axotomizing injury. Nonetheless, because the expression patterns of both fibronectin and laminin in cultured slices and perfused rat brain sections are similar, and DRGs in the in situ assay behaved similarly to neurons microtransplanted into white matter tracts in vivo, we speculate that the molecular and cellular environments encountered by the seeded neurons in our model are fundamentally similar to that in vivo.

Anti-fibronectin treatment of our slices caused a morphological change in callosal astrocytes not seen in those treated similarly on coverslips. It is likely that the mechanisms used by astrocytes to bind to the poly-lysine culture surface are different from those used to bind to each other in situ. In situ, astroglia may use fibronectin and its binding to astrocytic fibronectin receptors, including $\alpha 5 \beta 1$ and $\alpha \mathrm{v} \beta 8$ integrins (Tawil et al., 1993; Milner et al., 1999; King et al., 2001), to form focal adhesions with each other (Tawil et al., 1993; Takayama et al., 1999), providing a possible mechanism allowing for tract astrocyte alignment that may be disrupted by the anti-fibronectin antibody. Because oriented glia allow for an increased rate of neurite outgrowth (Biran et al., 2003), it is plausible that disruption of astrocytic focal contacts produces a disoriented terrain for regenerating neurites, resulting in a decrease in outgrowth. Thus, the question remains whether the antifibronectin-mediated effect on axon regeneration was caused, at least in part, by an indirect effect via changes in astrocyte shape. We had hoped to parse this out using antibodies that would specifically bind to mouse integrins located on the DRGs and not to rat integrins on the host astrocytes, but we were not able to obtain function-blocking antibodies. Of course, images of stubby neurites stopping short within a continuous astrocyte terrain suggest that anti-fibronectin did inhibit axon-glia interactions directly.

The abundant expression of fibronectin in white matter may be a contributing factor to the failure of axon regeneration by central neurons in the adult. We observed that although adult sensory neurons displayed robust outgrowth, adult cortical neurons did not grow well on slices containing white matter. It has been shown that CNS neurons can grow well on laminin (Rogers et al., 1983; Liesi et al., 1984) but not on fibronectin (Rogers et al., 1983). Sensory neurons, however, grow well on both substrates (Baron-Van Evercooren et al., 1982; Rogers et al., 1983). This dichotomy may be attributable to differences in the relative importance of the multiple binding domains in fibronectin for central versus sensory neurons. Central neurons bind fibronectin mainly via the heparin-binding site (Rogers et al., 1985, 1987; Haugen et al., 1992), whereas sensory neurons bind via both integrin- and heparin-binding sites (Rogers et al., 1985, 1987; Haugen et al., 1992; Lefcort et al., 1992; Yanagida et al., 1999; Vogelezang et al., 2001). Furthermore, it has also been suggested that a $10 \mathrm{kDa}$ peptide product of matrix metalloproteinase-2 (MMP-2)mediated digestion of the heparin-binding site actually inhibits cell attachment and outgrowth (Watanabe et al., 2000). Although MMP-2 is expressed by both central (Wang et al., 2002) and sensory neurons (Zuo et al., 1998) (our unpublished observations), central neurons may be affected more negatively by the 10 $\mathrm{kDa}$ peptide because they rely primarily on the heparin-binding site to mediate outgrowth on fibronectin. Therefore, the lack of laminin and presence of fibronectin in the mature corpus callosum, which may be a normal consequence of astrocyte aging (Smith et al., 1990), may further exacerbate the diminished intrinsic capacities of adult neurons to grow axons (Condic, 2001; Goldberg et al., 2002a,b). Thus, the limited adhesion molecule 

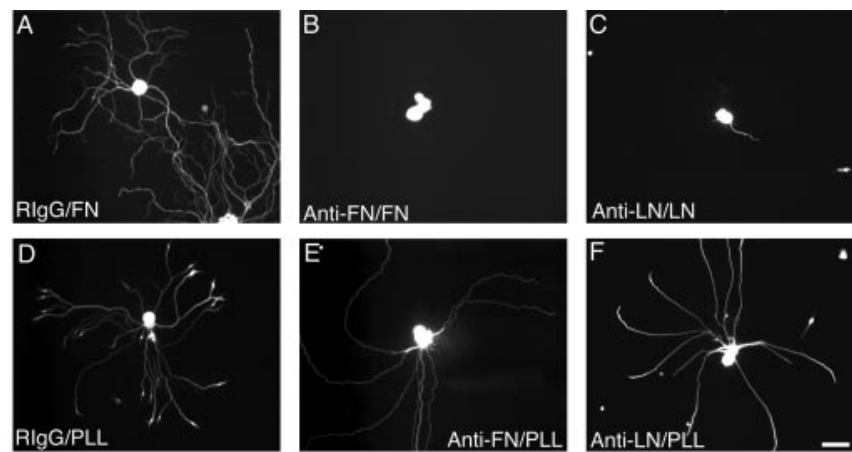

G

Neurite outgrowth on coverslips

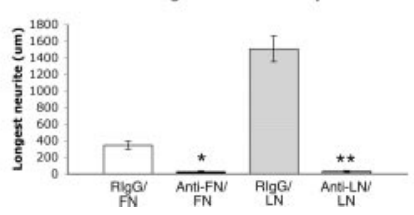

Figure 7. Polyclonal fibronectin (FN) and laminin (LN) antibodies are function blocking. $A-C, G$, Dissociated, adult DRGs were plated onto fibronectin-coated $(A, B)$ or laminin-coated coverslips $(C)$ and treated with control rabbit $\lg G(A)$, rabbit anti-fibronectin $(B)$, or rabbit anti-laminin $(C$ for $3 \mathrm{~d}$ and then stained for $\beta$-tubulin III. Although RlgG had no effect on neurite outgrowth, anti-fibronectin virtually abolished all outgrowth on fibronectin, and antilaminin greatly diminished outgrowth on laminin. The longest neurite of randomly chosen neurons on either FN- or LN-coated coverslips treated with either RlgG or the appropriate antibody ( $n=12$ per group) was measured and compared $(G)$. There was a statistical difference between the RlgG and anti-fibronectin treatments on neurites growing on fibronectin $\left({ }^{*} p<0.00001\right)$, and between RlgG and anti-laminin treatments on neurites growing on laminin $\left({ }^{* *} p<0.00000001\right) . D-F$, To control for antibody-mediated toxicity, adult DRGs were plated onto poly-lysine-coated coverslips and treated with $\operatorname{RlgG}(D)$, anti-fibronectin $(E)$, or anti-laminin $(F)$ for $3 \mathrm{~d}$. Neurons were able to extend long neurites with any of the three treatments, suggesting that the antibodies were not toxic, and both antibodies were able to specifically block outgrowth mediated by their respective molecules. Scale bar, $50 \mu \mathrm{m}$.

menu of the adult may pose a relatively larger impediment to the regeneration of CNS than sensory axons.

Interestingly, adult cortical neurons appeared to elaborate dendrites on the callosum. According to Goldberg et al. (2000a,b), the mature CNS neuron switches to a primarily dendrite-producing cell and focuses its transport mechanisms toward the production of dendrite-specific proteins. Also, Le Roux and Reh $(1995,1996)$ convincingly showed that, unlike axons, cortical dendrites growing on astrocytic monolayers were unaffected by both the maturity and reactive state of the astrocytes, in concurrence with our results. Although the mechanisms that underlie the curious difference between the growth properties of dendrites versus axons in a reactive glial environment are unknown, it may be attributable to a differential receptor repertoire or cytoskeletal transport capacity of the two types of processes.

The finding that sensory neurons are able to regenerate at all in white matter appears to challenge a great number of studies in the literature which suggest that myelin-related proteins potently inhibit outgrowth and regeneration (Caroni et al., 1988; Cadelli and Schwab, 1991; Fournier and Strittmatter, 2001; Filbin, 2003; Spencer et al., 2003); however, there are also several significant studies demonstrating that robust outgrowth in and on white matter is possible (Wictorin and Bjorklund, 1992; Davies et al., 1993, 1994, 1997, 1999; Li and Raisman, 1994; Pettigrew and Crutcher, 1999). How can these two contradictory sets of results be reconciled? First, it is important to note that although myelininduced growth cone collapse has been well documented (Fawcett et al., 1989; Bandtlow et al., 1990), it has also been shown that
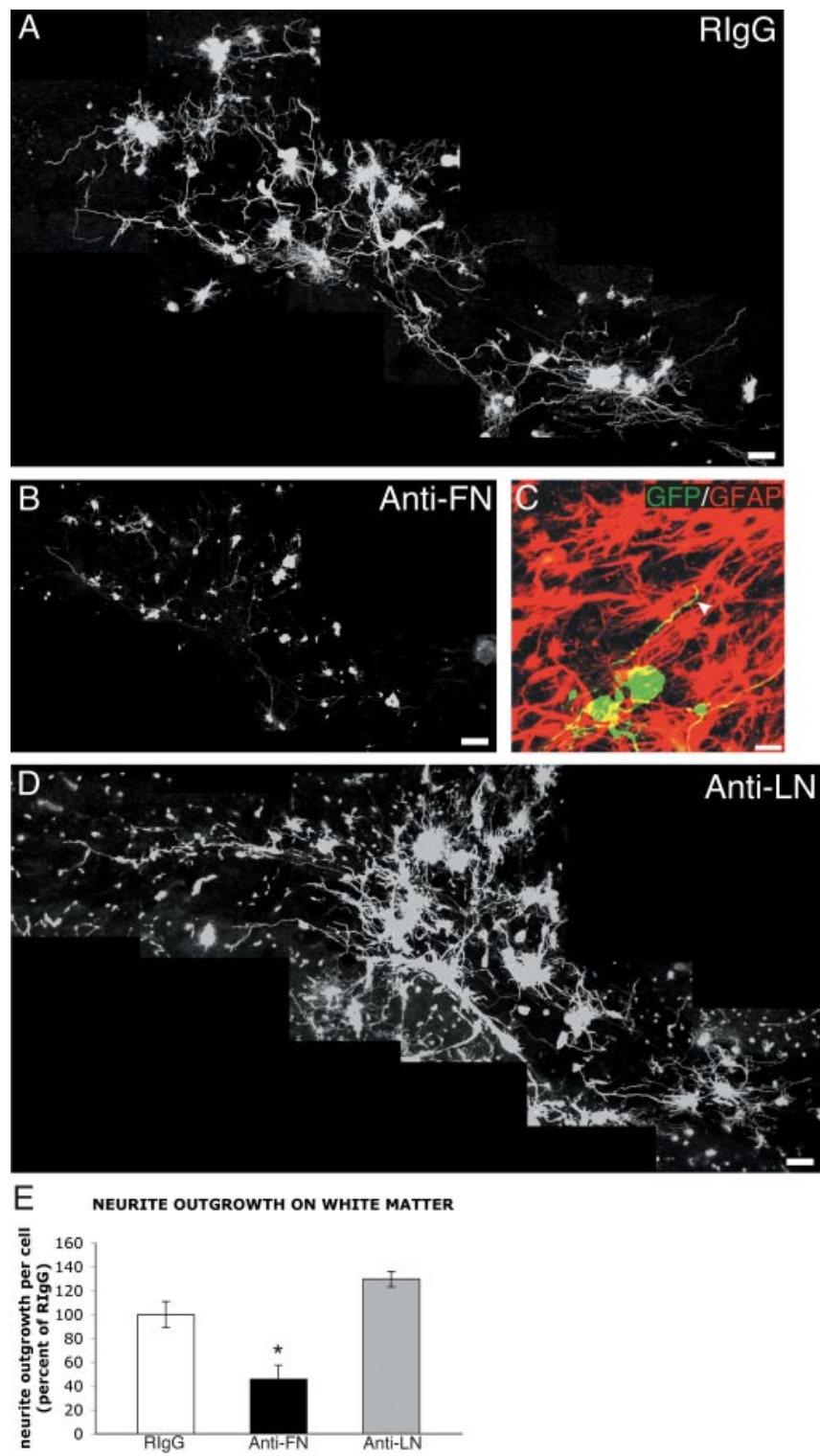

Figure 8. Anti-fibronectin, but not anti-laminin, decreases regeneration on white matter. After neuron seeding, the slice cultures were treated with control $\operatorname{Rlg} G(A)$, anti-fibronectin $(B)$, or anti-laminin ( $C$ ) for $3 \mathrm{~d}$. GFP immunohistochemistry is shown in $A, B$, and $D$. Blood vessels were also stained because the anti-rabbit secondary used to stain the GFP + neurites also recognized the anti-laminin and anti-fibronectin antibodies. Although there was extensive outgrowth along the corpus callosum in RlgG- and anti-laminin-treated slices, there was a diminished amount of outgrowth in anti-fibronectin-treated slices. C, High-magnification image of a fibronectin-treated slice double stained for GFP (green) and GFAP (red). Although the GFAP + astrocytes in the fibronectin-treated slices appeared smaller than in RlgG- or antilaminin-treated slices (compare with Figs. 2 and $5 A, B$ ), GFP + neurites (green) were not able to grow well even on an intact, longitudinal astrocytic terrain (arrowhead). $E$, Outgrowth on white matter of slices treated with RlgG $(n=10)$, anti-fibronectin $(n=10)$, or anti-laminin $(n=5)$ was digitally traced. The pixel areas of the tracings were quantified and statistically compared using a Student's $t$ test. Although there was no significant difference between RlgG- and antilaminin-treated slices, anti-fibronectin statistically decreased outgrowth when compared with the RlgG-treated controls ( $\left.{ }^{*} p<0.0001\right)$. These findings suggest that astrocyte-associated fibronectin plays a critical role in regeneration on the corpus callosum. Scale bars: $A, B, D, 100$ $\mu \mathrm{m} ; C, 25 \mu \mathrm{m}$.

oligodendrocyte inhibition can be overcome by the presence of astrocytes (Ard et al., 1991; Fawcett et al., 1992), laminin (David et al., 1995), and fibronectin (Caroni and Schwab, 1988), suggesting that the balance of environmental growth promotion and 
inhibition is being summed by the growth cone. Second, Pettigrew and Crutcher (1999) demonstrated that although the corpus callosum in cryosections inhibited the outgrowth of neurons seeded on the surface, the inhibition was critically dependent on the geometry of the white matter tract. When sections that had been cut perfectly tangentially to the longitudinal axis of the callosum were used, neurons sent out long, unbranched neurites parallel to the host tract. Does the ability of myelin to cause growth cone collapse help to generate parallel, nonbranched axon growth along astrocytes, rather than frank regeneration failure? Indeed, it has been hypothesized recently that myelin may play such a guidance function (Raisman, 2004). Growth cone filopodia that contact the highly oriented myelin may collapse transiently, and on recovery, change direction to grow unimpeded, parallel to the white matter, so long as there is also a strong growth-promoting substrate available. This theory is supported by the finding that neurons seeded on cryosections containing the corpus callosum from a myelin-deficient mouse extended neurites that branched repeatedly (Pettigrew and Crutcher, 2001). Disruption of the precise longitudinal arrangement of myelin may explain why neurite outgrowth was only roughly parallel with the callosal astrocytes in our slice model. In conclusion, if we hope to promote successful regeneration in the future, it is important to understand not only the growth-inhibiting molecules expressed after injury (Silver and Miller, 2004), but also what molecules are expressed (and conversely, not expressed) in white matter that may be exploited to help promote regeneration.

\section{References}

Adachi K, Yamauchi K, Bernaudin JF, Fouret P, Ferrans VJ, Crystal RG (1988) Evaluation of fibronectin gene expression by in situ hybridization: differential expression of the fibronectin gene among populations of human alveolar macrophages. Am J Pathol 133:193-203.

Ard MD, Bunge MB, Wood PM, Schachner M, Bunge RP (1991) Retinal neurite growth on astrocytes is not modified by extracellular matrix, anti-L1 antibody, or oligodendrocytes. Glia 4:70-82.

Asher RA, Morgenstern DA, Fidler PS, Adcock KH, Oohira A, Braistead JE, Levine JM, Margolis RU, Rogers JH, Fawcett JW (2000) Neurocan is upregulated in injured brain and in cytokine-treated astrocytes. J Neurosci 20:2427-2438.

Bandtlow C, Zachleder T, Schwab ME (1990) Oligodendrocytes arrest neurite growth by contact inhibition. J Neurosci 10:3837-3848.

Baron-Van Evercooren A, Kleinman HK, Ohno S, Marangos P, Schwartz JP, Dubois-Dalcq ME (1982) Nerve growth factor, laminin, and fibronectin promote neurite growth in human fetal sensory ganglia cultures. J Neurosci Res 8:179-193.

Biran R, Noble MD, Tresco PA (2003) Directed nerve outgrowth is enhanced by engineered glial substrates. Exp Neurol 184:141-152.

Braga de Souza S, Lent R (2004) Temporal and spatial regulation of chondroitin sulfate, radial glial cells, growing commissural axons, and other hippocampal efferents in developing hamsters. J Comp Neurol 468:217-232.

Brewer GJ (1997) Isolation and culture of adult rat hippocampal neurons. J Neurosci Methods 71:143-155.

Brown LF, Dubin D, Lavigne L, Logan B, Dvorak HF, Van de Water L (1993) Macrophages and fibroblasts express embryonic fibronectins during cutaneous wound healing. Am J Pathol 142:793-801.

Butler CD, Schnetz SA, Yu EY, Davis JB, Temple K, Silver J, Malouf AT (2004) Keratan sulfate proteoglycan phosphacan regulates mossy fiber outgrowth and regeneration. J Neurosci 24:462-473.

Cadelli DS, Schwab ME (1991) Myelin-associated inhibitors of neurite outgrowth and their role in CNS regeneration. Ann NY Acad Sci 633:234-240.

Caroni P, Schwab ME (1988) Two membrane protein fractions from rat central myelin with inhibitory properties for neurite growth and fibroblast spreading. J Cell Biol 106:1281-1288.

Caroni P, Savio T, Schwab ME (1988) Central nervous system regeneration: oligodendrocytes and myelin as non-permissive substrates for neurite growth. Prog Brain Res 78:363-370.
Condic ML (2001) Adult neuronal regeneration induced by transgenic integrin expression. J Neurosci 21:4782-4788.

David S, Braun PE, Jackson DL, Kottis V, McKerracher L (1995) Laminin overrides the inhibitory effects of peripheral nervous system and central nervous system myelin-derived inhibitors of neurite growth. J Neurosci Res 42:594-602.

Davies SJ, Fitch MT, Memberg SP, Hall AK, Raisman G, Silver J (1997) Regeneration of adult axons in white matter tracts of the central nervous system. Nature 390:680-683.

Davies SJ, Goucher DR, Doller C, Silver J (1999) Robust regeneration of adult sensory axons in degenerating white matter of the adult rat spinal cord. J Neurosci 19:5810-5822.

Davies SJA, Field PM, Raisman G (1993) Long fibre growth by axons of embryonic mouse hippocampal neurons micro-transplanted into the adult rat fimbria. Eur J Neurosci 5:95-106.

Davies SJA, Field PM, Raisman G (1994) Long interfascicular axon growth from embryonic neurons transplanted into adult myelinated tracts. J Neurosci 14:1596-1612.

Donaldson DJ, Mahan JT, Hasty DL, McCarthy JB, Furcht LT (1985) Location of a fibronectin domain involved in newt epidermal cell migration. J Cell Biol 101:73-78.

Dreyfus CF, Xudong D, Lercher LD, Racey BR, Friedman WJ, Black IB (1999) Expression of neurotrophins in the adult spinal cord in vivo. J Neurosci Res 56:1-7.

Fawcett JW, Rokos J, Bakst I (1989) Oligodendrocytes repel axons and cause axonal growth cone collapse. J Cell Sci 92:93-100.

Fawcett JW, Fersht N, Housden L (1992) Axonal growth on astrocytes is not inhibited by oligodendrocytes. J Cell Sci 103:571-579.

Filbin MT (2003) Myelin-associated inhibitors of axonal regeneration in the adult mammalian CNS. Nat Rev Neurosci 4:1-11.

Fitch MT, Doller C, Combs CK, Landreth GE, Silver J (1999) Cellular and molecular mechanisms of glial scarring and progressive cavitation: in vivo and in vitro analysis of inflammation-induced secondary injury after CNS trauma. J Neurosci 19:8182-8198.

Fournier AE, Strittmatter SM (2001) Repulsive factors and axon regeneration in the CNS. Curr Opin Neurobiol 11:89-94.

Gates MA, Fillmore H, Steindler DA (1996) Chondroitin sulfate proteoglycan and tenascin in the wounded adult mouse neostriatum in vitro: dopamine neuron attachment and process outgrowth. J Neurosci 16:8005-8018.

Goldberg JL, Espinosa JS, Xu Y, Davidson N, Kovacs GTA, Barres BA (2002a) Retinal ganglion cells do not extend axons by default: promotion by neurotrophic signaling and electrical activity. Neuron 33:689-702.

Goldberg JL, Klassen MP, Hua Y, Barres BA (2002b) Amacrine-signaled loss of intrinsic axon growth ability by retinal ganglion cells. Science 296:1860-1864.

Gonzalez ML, Malemud CJ, Silver J (1993) Role of astroglial extracellular matrix in the formation of rat olfactory bulb glomeruli. Exp Neurol 123:91-105.

Grimpe B, Dong S, Doller C, Temple K, Malouf AT, Silver J (2002) The critical role of basement membrane-independent laminin $\gamma 1$ chain during axon regeneration in the CNS. J Neurosci 22:3144-3160.

Guo Y, Martinez-Williams C, Yellowley CE, Donahue HJ, Rannels DE (2001) Connexin expression by alveolar epithelial cells is regulated by extracellular matrix. Am J Physiol 280:L191-L202.

Hafidi A, Lanjun G, Sanes DH (1999) Age-dependent failure of axon regeneration in organotypic culture of gerbil auditory midbrain. J Neurobiol 41:267-280.

Haugen PK, McCarthy JB, Roche KF, Furcht LT, Letourneau PC (1992) Central and peripheral neurite outgrowth differs in preference for heparin-binding versus integrin-binding sequences. J Neurosci 12:2034-2042.

Jones LL, Margolis RU, Tuszynski MH (2003) The chondroitin sulfate proteoglycans neurocan, brevican, phosphocan, and versican are differentially regulated following spinal cord injury. Exp Neurol 182:399-411.

King VR, McBride A, Priestley JV (2001) Immunohistochemical expression of the $\alpha 5$ integrin subunit in the normal adult rat central nervous system. J Neurocytol 30:243-252.

Lefcort F, Venstrom K, McDonald JA, Reichart LF (1992) Regulation of expression of fibronectin and its receptor, $\alpha 5 \beta 1$, during development and regeneration of peripheral nerve. Development 116:767-782. 
Le Roux PD, Reh TA (1995) Independent regulation of primary dendritic and axonal growth by maturing astrocyte in vitro. Neurosci Lett 198:5-8.

Le Roux PD, Reh TA (1996) Reactive astroglia support primary dendritic but not axonal outgrowth from mouse cortical neurons in vitro. Exp Neurol 137:49-65.

Li Y, Raisman G (1994) Long axon growth from embryonic neurons transplanted into myelinated tracts of the adult rat spinal cord. Brain Res 629:115-127.

Liesi P (1985) Laminin-immunoreactive glia distinguish regenerative adult CNS systems from non-regenerative ones. EMBO J 4:2505-2511.

Liesi P, Silver J (1988) Is astrocyte laminin involved in axon guidance in the mammalian CNS? Dev Biol 130:774-785.

Liesi P, Dahl D, Vaheri A (1983) Laminin is produced by early rat astrocytes in primary culture. J Cell Biol 96:920-924.

Liesi P, Dahl D, Vaheri A (1984) Neurons cultured from developing rat brain attach and spread preferentially to laminin. J Neurosci Res 11:241-251.

Liesi P, Kirkwood T, Vaheri A (1986) Fibronectin is expressed by astrocytes cultured from embryonic and early postnatal rat brain. Exp Cell Res 163:175-185

Matthiessen HP, Schmalenbach C, Müller HW (1989) Astroglia-released neurite growth-inducing activity for embryonic hippocampal neurons is associated with laminin bound in a sulfated complex and free fibronectin. Glia 2:177-188.

Milner R, Huang X, Wu J, Nishimura S, Pytela R, Sheppard D, ffrenchConstant C (1999) Distinct roles for astrocyte $\alpha v \beta 5$ and $\alpha v \beta 8$ integrins in adhesion and migration. J Cell Sci 112:4271-4279.

Moursi AM, Damsky CH, Lull J, Zimmerman D, Doty SB, Aota SI, Globus RK (1996) Fibronectin regulates calvarial osteoblast differentiation. J Cell Sci 109:1369-1380.

Müller HW, Junghans U, Kappler J (1995) Astroglial neurotrophic and neurite-promoting factors. Pharmacol Ther 65:1-18.

Neugebauer KM, Tomaselli KJ, Lilien J, Reichardt LF (1988) N-cadherin, NCAM, and integrins promote retinal neurite outgrowth on astrocytes in vitro. J Cell Biol 107:1177-1187.

Noble M, Fok-Seang J, Cohen J (1984) Glia are a unique substrate for the in vitro growth of central nervous system neurons. J Neurosci 4:1892-1903.

Pasinetti GM, Nichols NR, Tocco G, Morgan T, Laping N, Finch CE (1993) Transforming growth factor beta 1 and fibronectin messenger RNA in rat brain: responses to injury and cell-type localization. Neuroscience 54:893-907.

Pettigrew DB, Crutcher KA (1999) White matter of the CNS supports or inhibits neurite outgrowth in vitro depending on geometry. J Neurosci 19:8358-8366.

Pettigrew DB, Crutcher KA (2001) Myelin contributes to the parallel orientation of axonal growth on white matter in vitro. BMC Neurosci 2:9.

Pires-Neto MA, Braga de Souza S, Lent R (1998) Molecular tunnels and boundaries for growing axons in the anterior commissure of hamster embryos. J Comp Neurol 399:176-188.

Price J, Hynes RO (1985) Astrocytes in culture synthesize and secrete a variant form of fibronectin. J Neurosci 5:2205-2211.

Raisman G (2004) Myelin inhibitors: does NO mean GO? Nat Rev Neurosci 5:157-161.

Rogers SL, Letourneau PC, Palm SL, McCarthy J, Furcht LT (1983) Neurite extension by peripheral and central nervous system neurons in response to substratum-bound fibronectin and laminin. Dev Biol 98:212-220.

Rogers SL, McCarthy JB, Palm SL, Furcht LT, Letourneau PC (1985) Neuron-specific interactions with two neurite-promoting fragments of fibronectin. J Neurosci 5:369-378.

Rogers SL, Letourneau PC, Peterson BA, Furcht LT, McCarthy JB (1987)
Selective interaction of peripheral and central nervous system cells with two distinct cell-binding domains of fibronectin. J Cell Biol 105:1435-1442.

Rogers SL, Letourneau PC, Pech IV (1989) The role of fibronectin in neural development. Dev Neurosci 11:248-265.

Schwartz JP, Nishiyama N (1994) Neurotrophic factor gene expression in astrocytes during development and following injury. Brain Res Bull 35:403-407.

Silver J, Miller JH (2004) Regeneration beyond the glial scar. Nat Rev Neurosci 5:146-156.

Silver J, Edwards MA, Levitt P (1993) Immunocytochemical demonstration of early appearing astroglial structures that form boundaries and pathways along axon tracts in the fetal brain. J Comp Neurol 328:415-436.

Smith GM, Rutishauser U, Silver J, Miller RH (1990) Maturation of astrocytes in vitro alters the extent and molecular basis of neurite outgrowth. Dev Biol 138:377-390.

Snow DM, Lemmon V, Carrino DA, Caplan AI, Silver J (1990) Sulfated proteoglycans in astroglial barriers inhibit neurite outgrowth in vitro. Exp Neurol 109:111-130.

Spencer T, Domeniconi M, Cao Z, Filbin MT (2003) New roles for old proteins in adult CNS axonal regeneration. Curr Opin Neurobiol 13:133-139.

Steindler DA (1993) Glial boundaries in the developing nervous system. Annu Rev Neurosci 16:445-470.

Stewart GR, Pearlman AL (1987) Fibronectin-like immunoreactivity in the developing cerebral cortex. J Neurosci 7:3325-3333.

Takayama Y, Tanaka S, Nagai K, Okada M (1999) Adenovirus-mediated overexpression of C-terminal Src kinase (Csk) in type I astrocytes interferes with cell spreading and attachment to fibronectin. J Biol Chem 274:2291-2297.

Tawil N, Wilson P, Carbonetto S (1993) Integrins in point contacts mediate cell spreading factors that regulate integrin accumulation in point contacts vs. focal contacts. J Cell Biol 120:261-271.

Tom VJ, Steinmetz MP, Miller JH, Doller CM, Silver J (2004) Studies on the development and behavior of the dystrophic growth cone, the hallmark of regenerative failure, in an in vitro model of the glial scar and after spinal cord injury. J Neurosci 24:6531-6539.

Tomaselli KJ, Neugebauer KM, Bixby JL, Lilien J, Reichardt LF (1988) N-cadherin and integrins: two receptor systems that mediate neuronal process outgrowth on astrocyte surfaces. Neuron 1:33-43.

Vogelezang MG, Liu Z, Relvas JB, Raivich G, Scherer SS, ffrench-Constant C (2001) $\alpha 4$ integrin is expressed during peripheral nerve regeneration and enhances neurite outgrowth. J Neurosci 21:6732-6744.

Wang X, Mori T, Jung J, Fini ME, Lo EH (2002) Secretion of matrix metalloproteinase-2 and -9 after mechanical trauma injury in rat cortical cultures and involvement of MAP kinase. J Neurotrauma 19:615-625.

Watanabe K, Takahashi H, Habu Y, Kamiya-Kubushiro N, Kamiya S, Nakamura H, Yajima H, Ishii T, Katayama T, Miyazaki K, Fukai F (2000) Interaction with heparin and matrix metalloproteinase 2 cleavage expose a cryptic anti-adhesive site of fibronectin. Biochemistry 39:7138-7144.

Wictorin K, Bjorklund A (1992) Axon outgrowth from grafts of human embryonic spinal cord in the lesioned adult rat spinal cord. NeuroReport 3:1045-1048.

Yanagida H, Tanaka J, Maruo S (1999) Immunocytochemical localization of a cell adhesion molecule, interin $\alpha 5 \beta 1$, in nerve growth cones. J Orthop Sci 4:353-360.

Zuo J, Ferguson TA, Hernandez YJ, Stetler-Stevenson WG, Muir D (1998) Neuronal matrix metalloproteinase- 2 degrades and inactivates a neuriteinhibiting chondroitin sulfate proteoglycan. J Neurosci 18:5203-5211. 